\title{
Aspectos erotéticos del «hibridismo» de Mendel
}

\author{
Erotetic aspects of Mendel's «hibrydism»
}

\author{
PABLO LORENZANO \\ Universidad Nacional de Quilmes (UNQ)/ Consejo Nacional de Investigaciones \\ Científicas y Técnicas (CONICET) (Argentina)
}

Recibido: 16-1-2012

Aprobado definitivamente: 20-2-2013

\section{RESUMEN}

El objetivo de este trabajo es mostrar, en la línea sugerida por Nickles $(1980,1981)$ y desarrollada por Sintonen $(1985,1996)$, no sólo que el «enfoque de resolución de problemas» y el «enfoque de teorías» no son contrapuestos, sino que este último, mediante la versión de la concepción semántica de las teorías conocida bajo el nombre de «estructuralismo metateórico», puede ser utilizado para aportar precisión al enfoque de resolución de problemas, a través de la caracterización más precisa del contexto teórico en el que se plantean los problemas y, de este modo, de su individuación e historia, pudiéndose así distinguir dos tipos de «cambio problemático»: «cambio en un problema» y «cambio de problema». Para ello, se presentará dicha propuesta y luego será aplicada al caso del «hibridismo» de Mendel.

PALABRAS CLAVE

PROBLEMA CIENTÍFICO, ENFOQUE DE RESOLUCIÓN DE PROBLEMAS, ESTRUCTURALISMO METATEÓRICO, HIBRIDISMO

\footnotetext{
ABSTRACT

The aim of this paper is to show, in the line suggested by Nickles $(1980,1981)$ and developed by Sintonen $(1985,1996)$, not just that the «problem-solving approach» and the «theory approach» are not incompatible, but also that the latter, in the version of the semantic conception of theories known as «structuralist view», can be used to give precision to the problem-solving approach, 
by a more precise characterization of the theoretical context in which problems arise and, in this way, to their individuation and history, distinguishing two types of «problem change»: «change in a problem» and «change of a problem». In order to do this, it will be presented a proposal that will be applied to Mendel's «hybridism».

KEYWORDS

SCIENTIFIC PROBLEM, PROBLEM-SOLVING APPROACH. METATHEORETICAL STRUCTURALISM, HYBRIDISM

\section{INTRODUCCIÓN}

El OBJETIVO DE ESTE TRABAJO ES MOSTRAR no sólo que el «enfoque de resolución de problemas» y el «enfoque de teorías» no son contrapuestos, sino que este último, mediante la versión de la concepción semántica de las teorías conocida bajo el nombre de «concepción estructuralista», «metateoría estructuralista», «estructuralismo metateórico» o «estructuralismo metacientífico», puede ser utilizado efectivamente para aportar precisión al enfoque de resolución de problemas, a través de la caracterización más precisa del contexto teórico en el que se plantean los problemas y, de este modo, de su individuación e historia, pudiendo así distinguir dos tipos de «cambio problemático»: «cambio en un problema» y «cambio de problema». Para ello, se presentará brevemente una propuesta, basada en la que efectúa Sintonen $(1985,1996)$-quien a su vez sigue el análisis y las sugerencias de Tuomela (1980) - y en algunas ideas de Hintikka (1981), y luego será aplicada al caso del «hibridismo» de Johann Gregor Mendel, con sus dos partes: la «teoría de Mendel sobre el desarrollo/evolución de los híbridos» y la «teoría de Mendel sobre la fundamentación celular del desarrollo/ evolución de los híbridos».

\section{LA PROPUESTA EROTÉTICO-TEÓRICA: SOBRE EL TRATAMIENTO ESTRUCTURALISTA DE LOS ASPECTOS EROTÉTICOS}

La idea básica de la propuesta erotético-teórica es la de combinar algunos aspectos de ciertos análisis erotéticos (de sistemas de lógica erotética, de enfoques de resolución de problemas, de modelos interrogativos, de preguntas y respuestas y de problemas y soluciones) con el análisis que el estructuralismo metacientífico realiza de las teorías científicas, en sus dimensiones sincrónica y diacrónica. De los distintos sistemas de lógica erotética y modelos interrogativos, por el momento nos quedaremos con la idea que es factible representar la forma lógica de una pregunta/enunciado interrogativo/problema mediante la combinación de ciertos enunciados declarativos, aseverativos, en el modo indi- 
cativo (o proposiciones) con algún operador, o cuantificador, de interrogación (ver p.e. Belnap \& Steel 1976).

Basándonos en gran medida en la propuesta de Sintonen $(1985,1996)$, quien a su vez sigue el análisis y las sugerencias de Tuomela (1980), podríamos decir que las teorías proveen lo que se han denominado presuposiciones del hablante $_{2}$ (en analogía con las que se han llamado presuposiciones ${ }_{1}$ de la lógica erotética, en terminología de Tuomela 1980) o bien presuposiciones relevantes o presuposiciones pragmáticas de preguntas (para marcar la diferencia con las presuposiciones de la lógica erotética, en terminología de Sintonen 1985), $\mathrm{y}$, de este modo, identificar un problema no con el contenido ilocucionario de un enunciado interrogativo, sino con su pregunta completa subyacente. Dicha pregunta puede ser representada mediante un par ordenado $Q=\langle(? p) q(p), r\rangle$, en donde (?p) $q(p)$ es la forma lógica del enunciado de la pregunta (siendo $p$ la presuposición lógica y $q$ la pregunta) y $r$ el conjunto de presuposiciones del

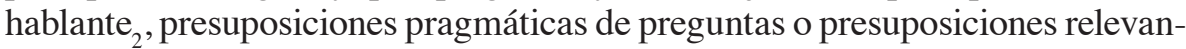
tes $_{2}$. Sin embargo, para que la pregunta completa subyacente sea identificada con precisión, debemos decir aún cuáles son las presuposiciones relevantes (los elementos del conjunto $r$ ) que incluiremos en ella. Para lo cual, siguiendo a Sintonen $(1985,1996)$, se recurrirá a la noción estructurada de teoría del estructuralismo metacientífico. Además, como veremos más adelante, las preguntas (y sus respuestas) también tienen una historia (susceptible de ser analizada a partir de una distinción realizada por Hintikka 1981), que va en algún sentido de la mano de la historia de las teorías.

De acuerdo, entonces, con la propuesta que combina los análisis erotéticos con el análisis estructuralista, el componente más básico para la identificación de las restricciones de los problemas (las presuposiciones del hablante ${ }_{2}$, presuposiciones pragmáticas de preguntas, o presuposiciones relevantes ${ }_{2}$ ) de un científico, miembro de una comunidad o generación científica, ${ }^{1}$ que acepta en un momento dado $t$ una teoría, es el denominado «paradigma kuhniano» $\left.\left\langle\mathbf{K}_{0}, \mathbf{I}\right\rangle_{e}\right\rangle$ con sus constituyentes: el núcleo básico $\mathbf{K}_{0}$, con el vocabulario y la «gramática» de la teoría (sus modelos potenciales $\mathbf{M}_{\mathbf{p}}$ y modelos parciales $\mathbf{M}_{\mathrm{pp}}$ ), la/s ley/es fundamental/es (sus modelos $\mathbf{M}$ ), las condiciones de ligadura (C) y los vínculos (L), y las aplicaciones paradigmáticas o ejemplares $\mathbf{I}_{e}$ (que, al proporcionar soluciones paradigmáticas a las preguntas previas, restringen las respuestas y le proveen una guía heurística al científico). ${ }^{2}$

1 Una generación científica puede verse como una comunidad científica considerada desde un punto de vista sincrónico (Balzer, Moulines \& Sneed 1987, § V.1.4; Moulines 1991, § III.4.2).

2 Para una presentación completa de las nociones estructuralistas, ver Balzer, Moulines \& Sneed (1987). 
De este modo, la pregunta completa para una generación de científicos que aplica una teoría en el tiempo $t$ tiene la forma $Q=\left\langle(? p) q(p),\left\langle\mathbf{K}_{0}, \mathbf{I} e\right\rangle\right.$.

Preguntas y respuestas pueden ser parafraseadas utilizando distintos vocabularios. Una pregunta puede concernir a un sistema, el cual es descrito en el lenguaje-en-uso de una comunidad científica y sólo recién más tarde ser descrito en el lenguaje de un elemento teórico establecido. Puede haber preguntas «porqué» que exijan explicación, expresadas en el lenguaje informal de la comunidad científica (quizás en el vocabulario con el que se formulan los $\mathbf{M}_{\mathrm{pp}}$ ), antes de que $\mathbf{M}_{\mathrm{p}}$ y $\mathbf{M}$ del elemento teórico $\mathbf{T}$ haya sido articulado. Y una vez que $\mathbf{M}_{\mathrm{p}}$ está disponible, uno puede plantear la pregunta ulterior de si las estructuras dentro del conjunto I, ahora enriquecidas con funciones teóricas, son además modelos. También puede haber preguntas «cómo» expresadas en el lenguaje informal de la comunidad científica, antes de que $\mathbf{M}_{\mathbf{p}}$ y $\mathbf{M}$ del elemento teórico $\mathbf{T}$ haya sido articulado. Para el caso de las teorías llamadas «fenomenológicas», que carecen de conceptos T-teóricos y en donde las estructuras que representan los modelos potenciales $\mathbf{M}_{\mathbf{p}}$ poseen el mismo tipo lógico de aquellas que representan los modelos parciales $\mathbf{M}_{\mathrm{pp}}$, una vez que $\mathbf{M}_{\mathrm{p}}$ está disponible, uno puede plantear la pregunta ulterior de si las estructuras dentro del conjunto $\mathbf{I}$ (en donde $\mathbf{I} \subseteq \mathbf{M}_{\mathrm{p}}$ ) son además modelos.

La investigación procede intentando refinar las preguntas encontrando el vocabulario adecuado para expresar las respuestas y explorando posibles leyes especiales (especializaciones) expresadas mediante ese vocabulario.

Una interrogación que comienza como una imprecisa pregunta «porqué», formulada en el lenguaje-en-uso de la comunidad científica (en el vocabulario T-no-teórico o, incluso, en el perteneciente a «pre-teorías», «proto-teorías» o «folk-theories» subyacentes), y concerniente a un área de experiencia («ipor qué $i$ ?», en donde $i$ es un fenómeno de algún tipo), se torna (en el estado «paradigmático» en el que hay un núcleo conceptual $\mathbf{K}_{0}$ con su vocabulario) en una pregunta «qué» («iqué leyes especiales se necesitan para gobernar este particular conjunto de aplicaciones $\mathbf{I}_{i} \subseteq \mathbf{I}$ ?») o «cuál» o «cuáles» (p.e., «¿cuál es la ley especial que rige este particular conjunto de aplicaciones $\mathbf{I}_{i}$ ?» $\mathrm{O}$ «icuáles son las especificaciones de los componentes teóricos apropiadas para dar cuenta de este particular conjunto de aplicaciones $\left.\mathbf{I}_{i} ? »\right)$, que incorpora el vocabulario T-teórico. Es una tarea ulterior la de encontrar preguntas «sí-no» (p.e. «ies ésta la ley especial que rige este particular conjunto de aplicaciones $\mathbf{I}_{i}$ ?» $\mathrm{O}$ «ison éstas las especificaciones de los componentes teóricos apropiadas para dar cuenta de este particular conjunto de aplicaciones $\mathbf{I}_{i}$ ?»), que delineen alternativas específicas y reduzcan aún más las respuestas admisibles. De manera análoga, en el caso de teorías fenomenológicas, una interrogación que comienza como una imprecisa pregunta «cómo», formulada en el lenguaje-en-uso de la comunidad científica, y concerniente a un área de experiencia («¿cómo $i$ ?», en donde 
$i$ es un fenómeno de algún tipo), se torna (en el estado «paradigmático» en el que hay un núcleo conceptual $\mathbf{K}_{0}$ con su vocabulario) en una pregunta «qué» («iqué leyes especiales se necesitan para gobernar este particular conjunto de aplicaciones $\mathbf{I}_{i} \subseteq \mathbf{I}$ ?») o «cuál» o «cuáles» (p. e., «¿cuál es la ley especial que rige este particular conjunto de aplicaciones $\mathbf{I}_{i}$ ?» $\mathrm{O}$ «icuáles son las especificaciones apropiadas para dar cuenta de este particular conjunto de aplicaciones $\mathbf{I}_{i}$ ?»). Es una tarea ulterior la de encontrar preguntas «sí-no» (p. e. «ies ésta la ley especial que rige este particular conjunto de aplicaciones $\mathbf{I}$ ? ? $\mathbf{0}$ 《ison éstas las especificaciones apropiadas para dar cuenta de este particular conjunto de aplicaciones $\mathbf{I}$ ? »), que delineen alternativas específicas y reduzcan aún más las respuestas admisibles.

Se explicitó qué es un problema al interior de una teoría en el sentido de Kuhn. Pero, ¿hay problemas comunes a distintas teorías? ¿Existe un tipo de inconmensurabilidad entre problemas análogo al de la inconmensurabilidad entre teorías? Y si esto es así, ¿no se podría decir algo similar a lo que ha sido dicho sobre la inconmensurabilidad entre teorías en el marco de la concepción estructuralista?

Esta es una posibilidad que nos muestra Hintikka (1981, pp. 79-80). Él dice que, para poder entender mejor el papel de los conceptos teóricos y de los marcos conceptuales, el marco (lingüístico) en el cual se formula una respuesta a una pregunta dada, no necesita ser completamente el mismo que aquél en el cual se formula la pregunta. El primero de los marcos puede contener conceptos adicionales. De allí que se deba distinguir entre presuposiciones para preguntas y presuposiciones para respuestas. Las presuposiciones del primer tipo contendrían sólo la parte no-teórica del marco conceptual, mientras que las del segundo tipo contendrían la totalidad (e.e. también la parte teórica) de tal marco.

La idea básica es muy sencilla: 1) uno puede formular las preguntas (plantear los problemas) que le permiten su «vocabulario»-eventualmente considerando la distinción entre conceptos T-teóricos y conceptos T-no-teóricos, o sea, entre los $\mathbf{M}_{\mathrm{p}}$ y los $\mathbf{M}_{\mathrm{pp}}$ (aun cuando en algunos casos se deba «descender» aún más en los niveles de presuposición, yendo hacia «pre-teorías», «proto-teorías» $\mathrm{o}$ «folk-theories» subyacentes)-, pudiendo utilizarlo a veces en su totalidad, a veces sólo en parte; 2) uno puede responder las preguntas (resolver los problemas) como se lo permite toda su «teoría», e. e. todo el marco conceptual, la/s ley/es fundamental/es (más quizás alguna(s) especial(es)), las condiciones de ligadura, los vínculos y las aplicaciones exitosas.

En el caso más simple, y simultáneamente típico, que no es el caso de las teorías «fenomenológicas», en que relacionamos la distinción entre presuposiciones lógicas (presuposiciones, o presuposiciones de preguntas) y presuposiciones pragmáticas de preguntas (presuposiciones del hablante ${ }_{2}$, presuposiciones 
relevantes ${ }_{2}$ o presuposiciones de respuestas), por un lado, con la distinción entre conceptos T-teóricos y conceptos T-no-teóricos, por el otro, podríamos considerar que las presuposiciones del primer tipo sólo contienen la parte T-noteórica de tal marco conceptual de la teoría, mientras que las presuposiciones del segundo tipo contienen la totalidad del marco conceptual, e.e. también la parte T-teórica de dicho marco.

Con ello, podemos distinguir entre dos tipos de «cambio problemático» - llamado así en analogía con el «cambio teórico»- (dependiente de teorías o paradigmas kuhnianos): 1) cambio en un problema, y 2) cambio de problema.

El primer tipo de cambio problemático o «cambio en un problema» es aquél en donde no hay cambio de las teorías o paradigmas kuhnianos que lo restringen, sino que es aquél en el que, en todo caso, tiene lugar un cambio intrateórico (e.e. una evolución teórica). Aquí podemos hablar de reformulaciones más precisas de uno y el mismo problema; tales reformulaciones presuponen pragmáticamente no sólo al paradigma kuhniano, sino también a los elementos teóricos precedentes dentro de la red teórica en la que se está trabajando en un momento de la evolución teórica, con las leyes especiales (y constricciones adicionales) de sus elementos teóricos predecesores.

El segundo tipo de cambio problemático o «cambio de problema» es aquél en donde sí hay un cambio de las teorías o paradigmas kuhnianos que lo restringen, e.e. en donde tiene lugar un cambio interteórico (que pudiera ser del tipo de una revolución científica). Aquí, aun cuando pudiera permanecer inalterable (aunque no necesariamente) el primero de los componentes (la forma lógica de la pregunta (?p) $q(p)$ ), el segundo de los componentes (el paradigma kuhniano $\left.\left.\left\langle\mathbf{K}_{0}, \mathbf{I}\right\rangle\right\rangle\right)$ cambia y, así, también la pregunta completa subyacente.

Como paso previo a la identificación de sus aspectos erotéticos, a continuación identificaremos los aspectos conceptuales de las teorías correspondientes al hibridismo de Mendel, e.e. a la «teoría de Mendel sobre el desarrollo/evolución de los híbridos» y a la «teoría de Mendel sobre la fundamentación celular del desarrollo/evolución de los híbridos».

\section{Aspectos CONCEPTUALES DEL «HIBRIDISMO» DE MENDEL ${ }^{3}$}

En el trabajo de Mendel (1865) se pueden diferenciar dos partes principales, o niveles, que corresponden a las dos máximas guías especiales de Schleiden: ${ }^{4}$

3 Para un análisis historiográfico más extenso de la obra de Mendel y su contexto, ver Lorenzano (1995, 1997, 2011).

4 Schleiden (1849), siguiendo la llamada «filosofía natural kantiana-friesiana»-también llamada «filosofía natural matemática» $\mathrm{y}$ «escuela friesiana»-fundada por Jakob Friederich Fries en Jena, reformuló especialmente para la botánica considerada como una «ciencia inductiva» las máximas guías («leitende Maximen») o principios regulativos («regulative Principien») 
una, en donde, como paso previo para poder resolver el problema de la hibridación («ipueden originarse nuevas especies a partir del cruzamiento de especies preexistentes?»), que para Mendel era el problema central en la historia evolutiva de los seres vivos, se propone encontrar «una ley de validez universal sobre la formación y la evolución de los híbridos», es decir, encontrar el modo en que los hibridos se desarrollan/evolucionan, lo cual realiza a partir de un análisis estadístico de sus experimentos, básicamente realizados con Pisum, y en donde formula «la ley de desarrollo/evolución encontrada en Pisum», que se descompone en «la ley de la combinación simple de las características» $\aleph^{5}$ y su generalización, «la ley de la combinación de las características diferenciales», esto es, una parte que se mueve a un nivel, digamos, más «empírico» o «fenomenológico» y que corresponde a la máxima de la historia del desarrollo/ evolución de Schleiden; ${ }^{7}$ otra, en la que intenta fundamentar y explicar «la ley de desarrollo/evolución encontrada en Pisum», que rige el comportamiento de los «híbridos variables», pero también la ley que regiría el comportamiento de los «híbridos constantes» -entre los cuales se encuentran, según Mendel (1865), Aquilegia atropurpureo-canadensis, Lavatera pseudolbio-thuringiaca, Geum urbano-rivale, algunos híbridos de Dianthus, los híbridos de las especies de sauces y, de acuerdo con Mendel (1869), Hieracium-, mediante la relación entre la producción y comportamiento de las células germinales y polínicas y el de las formas (o características) constantes (Mendel 1865, p. 32) y, en última instancia, en la naturaleza y comportamiento («constitución y agrupamiento») de lo que denomina «elementos» (Mendel 1865, p. 58) o «elementos celulares»

generales para la investigación de la naturaleza de Fries -acorde con las cuales las inducciones e hipótesis son orientadas, juzgadas y justificadas.

$5 \ll$ «i $A$ denota una de las características constantes, por ejemplo la dominante; $a$ denota la recesiva y Aa la forma híbrida, así da la expresión: $A+2 A a+a$ la serie de desarrollo/ evolución para los descendientes de los híbridos para dos características diferenciales» (Mendel 1865, pp. 34-35).

6 «[L]os descendientes de los híbridos en los cuales se han unido varias características esencialmente diferentes, presentan los miembros de una serie combinatoria en la que se han unido las series de desarrollo para dos características diferenciales. Con esto se demuestra, al mismo tiempo, que el comportamiento de cada dos (tipos de) características diferenciales es independiente en la unión híbrida de cualesquiera otras diferencias en las plantas parentales» (Mendel 1865, p. 40).

7 Schleiden (1849) enuncia la primera de las máximas guías del siguiente modo: «A. Máxima de la historia del desarrollo/evolución [Entwicklungsgeschichte]. [...] la única posibilidad de alcanzar una comprensión científica en la botánica, y así el único e ineludible instrumento que se origina por sí mismo en la naturaleza del objeto, es el estudio de la historia del desarrollo/ evolución [Entwicklungsgeschichte]. [...] toda hipótesis, toda inducción en la botánica que no esté orientada por la historia del desarrollolevolución [Entwicklungsgeschichte] debe rechazarse incondicionalmente» (Schleiden 1849, 141,142, 146; énfasis del autor). 
(Mendel 1865, p. 60), ya sea de una unión pasajera de los elementos celulares diferenciales para explicar el comportamiento de los híbridos variables o de una unión duradera de los elementos celulares diferenciales para explicar la existencia de híbridos constantes, esto es, una parte que se mueve a un nivel, digamos, más «teórico» y que corresponde a la máxima de la autonomía de las células de las plantas de Schleiden. ${ }^{8}$ En la primera parte, Mendel propone lo que se podría denominar la «teoría de Mendel sobre el desarrollo/evolución de los híbridos» (MEH), en tanto que, en la segunda, Mendel sugiere lo que se podría denominar la «teoría de Mendel sobre la fundamentación celular del desarrollo/evolución de los híbridos» (MFC). A continuación presentaremos un análisis estructuralista de ambas teorías. ${ }^{9}$

\section{III.1. LA TEORÍA DE MENDEL SOBRE EL DESARROLLO/EVOLUCIÓN DE LOS HÍBRIDOS} (MEH)

Comenzando con la «teoría de Mendel sobre el desarrollo/evolución de los híbridos» (MEH), su marco conceptual, o sea, la clase de sus modelos potenciales $\mathbf{M}_{\mathrm{p}}(\mathbf{M E H})$, se podría representar mediante estructuras como la siguiente: $\left\langle I,\left(C_{i}\right)_{i s k}, A P P, M A T, D I S T\right\rangle$, en donde $I$ representa el conjunto de individuos (progenitores y descendientes), que constituyen poblaciones vinculadas por relaciones de consanguineidad, denominadas «familias», $\left(C_{i}\right)_{i s k}$ el conjunto de tipos de características (en donde cada conjunto $C_{i}$ debería ser considerado como una característica y los elementos $c_{i} \in C_{i}$ como rasgos o expresiones de esa característica), $A P P$ una función que le asigna a los individuos sus rasgos o características de cierto tipo o apariencia, MAT una función de cruza que le asigna a dos padres cualesquiera su descendencia y DIST las frecuencias relativas de los rasgos o características de cierto tipo observadas en la descendencia.

Los cuatro primeros de estos conceptos no son ajenos a las tradiciones de los «criadores» y de los «hibridistas»; el que sí es «novedoso» es el que representa el análisis estadístico que lleva a cabo de los cruzamientos y proporciona las frecuencias relativas de los rasgos o características de cierto tipo observadas en la descendencia: la función DIST, que describe la transición de las características de los progenitores a distribuciones de características en la descendencia. Sin embargo, a pesar de ser «novedoso», o sea, no «disponible con

8 La segunda de las máximas guías para la botánica es formulada por Schleiden del siguiente modo: «B. Máxima de la autonomía de las células de las plantas. [...] en lo esencial la vida de las plantas debe estar contenida en la vida de las células [...] toda hipótesis, toda inducción que no apunte a explicar los procesos que ocurren en la planta como resultado en los cambios que tienen lugar en las células individuales debe rechazarse incondicionalmente» (Schleiden 1849, 146, 148; énfasis del autor).

9 Para un análisis estructuralista alternativo de lo que allí se denomina «la Teoría de la Hibridación de Mendel», ver Casanueva (2002). 
anterioridad»y, en ese sentido, «teórico», de acuerdo con el criterio «histórico» de Hempel (1966, pp. 73-75), si aplicamos el criterio usual de T-teoricidad del estructuralismo (ver p. e. Balzer, Moulines \& Sneed 1987, pp. 47-73, 391-393), más «sistemático» que el de Hempel, según el cual un término es T-teórico si todos los métodos de determinación (de la extensión del concepto expresado por el término) dependen de $\mathbf{T}$, o sea, son $\mathbf{T}$-dependientes, presuponiendo directa o indirectamente la validez de las leyes de $\mathbf{T}$; si alguno no la presupone, e.e. si se puede determinar independientemente de $\mathbf{T}$, el término es $\mathbf{T}$-no-teórico, la función DIST es un término T-no-teórico para la teoría $\mathbf{M E H}$, es decir, MEH-no-teórico, pues la determinación de la frecuencia relativa de los rasgos o características observadas en la descendencia no depende de MEH; más bien, basta contar tanto el número total $n$ de la descendencia como el número $m_{i}$ de individuos con la característica de ese tipo en la descendencia para determinar la frecuencia relativa de la ocurrencia de esa característica particular $r_{i}$, que se obtiene dividiendo $m_{i}$ entre $n$; si juntamos todas esas frecuencias relativas, se obtiene la distribución o frecuencia relativa de características en la descendencia. ${ }^{10}$ Así, vemos que, aunque la novedad de un concepto sea por lo general indicativo de la plausibilidad prima facie de considerar dicho concepto como teórico para la teoría $\mathbf{T}$ en donde se lo introduce por vez primera, los criterios «histórico» de Hempel y «sistemático» estructuralista no deben coincidir necesariamente.

Al no encontrar conceptos MEH-teóricos, dicha teoría es fenomenológica en el sentido anteriormente señalado: en ella, no sólo los modelos potenciales $\mathbf{M}_{\mathbf{p}}(\mathbf{M E H})$ son del tipo lógico indicado, sino que también sus modelos parciales $\mathbf{M}_{\mathrm{pp}}^{\mathbf{p}}(\mathbf{M E H})$ poseen ese mismo tipo lógico: $\left\langle I,\left(C_{i}\right)_{i \leq k}, A P P, M A T, D I S T\right\rangle, \mathrm{y}$ en donde los distintos componentes se interpretan del modo ya indicado.

Podemos suponer que los conceptos que ocurren en las estructuras de ese tipo lógico serían los utilizados para expresar la «ley de validez universal sobre la formación y la evolución de los híbridos», al igual que la ley que regiría el desarrollo/evolución de los híbridos constantes, ya que son los utilizados para expresar la ley que rige el desarrollo/evolución de los híbridos variables, «la ley encontrada en Pisum». Mendel reconoce que «que no poseemos una teoría completa de la hibridación» (Mendel 1869, p. 28). De la red teórica que conforma dicha teoría, él llegó a proponer de manera desarrollada sólo un elemento teórico especializado, el que representa la línea de especialización correspondiente

10 Más aún, en la medida en que dicha distribución se determina unívocamente por medio del número total de la descendencia y del número de la descendencia que muestra las características en cuestión, es decir, por medio de las funciones MAT y APP, la función DIST puede ser considerada como definible en función de las anteriores y, de este modo, como un concepto no-primitivo. 
al desarrollo/evolución de los híbridos variables (MEHV), insinuando en qué consistiría el elemento teórico especializado que representaría la línea de especialización correspondiente al desarrollo/evolución de los híbridos constantes (MEHC) y sólo sugiriendo la existencia del elemento teórico básico $\left(\mathbf{M E H}_{0}\right)$, que contendría «una ley general más alta» (Mendel 1870, p. 1270), la «ley de validez universal sobre la formación y la evolución de los híbridos», que sería la ley fundamental de $\mathbf{M E H}$, pero que Mendel nunca alcanzó a formular. ${ }^{11}$ Los distintos sistemas empíricos de tipo $\left\langle I,\left(C_{i}\right)_{i \leq k}, A P P, M A T, D I S T\right\rangle$ que satisficieran esta ley, así como la insinuada ley sobre el desarrollo/evolución de los híbridos constantes o la planteada ley sobre el desarrollo/evolución de los híbridos variables, constituirían la clase de los modelos de MEH (M(MEH)).

A pesar de que MEH carece, según vimos, de términos MEH-teóricos, de todos modos parece que podemos identificar en ella una condición de ligadura. Ella establece una relación del tipo de las denominadas de igualdad, a saber: la exigencia de que los mismos tipos de características parentales se distribuyen en la descendencia siempre de la misma manera en todas las aplicaciones de la teoría de Mendel sobre el desarrollo/evolución de los híbridos en que ellos ocurran, o sea, es una condición de ligadura sobre la función DIST (en símbolos: $\left.\mathbf{C}_{D I S T}^{\square,}\right)$. Así, $\mathbf{C}(\mathbf{M E H}):=\mathbf{C}_{D I S T}^{\square,}$.

Los vínculos que MEH tiene con otras teorías (pre-teorías, proto-teorías, «folk-theories») subyacentes, presupuestas, por medio de las cuales se determina la extensión de aquellos conceptos que son MEH-no-teóricos, o sea, de los conceptos simbolizados como $I,\left(C_{i}\right)_{i s k}, A P P, M A T$ y DIST son los siguientes. Para determinar la extensión del concepto de individuo (biológico) $I$ bastaría alguna «teoría» proveniente del conocimiento común (simbolicemos este vínculo por medio de $\lambda_{1}$ ), permitiendo establecer la «interpretación intencional básica» de este conjunto base principal. Eventualmente, lo mismo ocurriría con el concepto de rasgo o tipo de característica $\left(C_{i}\right)_{i s k}$ y con el de apariencia $A P P$; en caso de que no bastara cualquier «teoría» proveniente del conocimiento común, podría llegar a hacerlo con una un poco más sofisticada, del tipo de la que ya encontramos en posesión de los criadores de animales, cultivadores de plantas e hibridistas de especies hacia fines del siglo XVIII (simbolicemos este vínculo mediante $\lambda_{2}$ ). A través del concepto de cruzamiento de individuos que dejan descendencia MAT, el mendelismo se vincula con alguna teoría de la reproducción biológica (y, a través de ella, con la teoría celular), aun cuando ésta no sea demasiado elaborada o detallada (simbolicemos este vínculo por

11 «No puedo en esta ocasión reprimir la observación de cómo debe ser llamativo que los híbridos de Hieracium observan, en comparación con los de Pisum, un comportamiento directamente opuesto. Tenemos que tratar aquí evidentemente sólo con fenómenos aislados, que son el resultado de una ley general más alta.» (Mendel 1870, p. 1270). 
medio de $\lambda_{3}$ ). Por último, el mendelismo, mediante el concepto de distribución (estadística) de las características en la descendencia DIST, que, recordemos, no es un auténtico concepto primitivo, sino que es definible en términos de las funciones $A P P$ y $M A T$, se vincula con las teorías de la reproducción biológica y del conocimiento común sofisticado poseído por los criadores de animales, cultivadores de plantas e hibridistas de especies de fines del siglo XVIII con las que se relacionan tales funciones, pero, además, lo hace con la teoría estadística (simbolicemos este vínculo mediante $\lambda_{4}$ ). El vínculo interteórico global de MEH (L(MEH)), formado por la intersección de todos vínculos interteóricos que tiene esta teoría con otras teorías subyacentes, presupuestas, se define de la siguiente manera: $\mathbf{L}(\mathbf{M E H}):=\cap\left\{\lambda_{1}, \lambda_{2}, \lambda_{3}, \lambda_{4}\right\}$.

Si dispusiéramos de una teoría completa sobre el desarrollo/evolución de los híbridos, estaríamos ahora en condiciones de caracterizar su núcleo teórico $\left(\mathbf{K}_{0}(\mathbf{M E H})\right)$ como sigue:

$$
\mathbf{K}_{0}(\mathbf{M E H}):=\left\langle\mathbf{M}_{\mathbf{p}}(\mathrm{MEH}), \mathbf{M}(\mathrm{MEH}), \mathbf{M}_{\mathrm{pp}}(\mathrm{MEH}), \mathbf{C}(\mathrm{MEH}), \mathbf{L}(\mathbf{M E H})\right\rangle .
$$

El dominio de aplicaciones intencionales de MEH está constituido por la clase de aquellos sistemas empíricos a los que uno desearía aplicar lo que sería su ley fundamental, la «ley de validez universal sobre la formación y la evolución de los híbridos». Lo único que podemos decir desde un punto de vista formal es que una aplicación propuesta es un modelo potencial o, lo que es lo mismo en este caso, un modelo parcial. Esto significa que $\mathbf{I}(\mathbf{M E H}) \subseteq \mathbf{M}_{\mathbf{p}}(\mathbf{M E H})$ (o que $\mathbf{I}(\mathbf{M E H}) \subseteq \mathbf{M}_{\mathrm{pp}}(\mathbf{M E H})$, pues $\left.\mathbf{M}_{\mathrm{p}}(\mathbf{M E H})=\mathbf{M}_{\mathrm{pp}}(\mathbf{M E H})\right)$ y que los miembros de I(MEH) son sistemas empíricos que contienen individuos (individuos propiamente dichos o poblaciones) con una cierta apariencia (es decir, con ciertos rasgos o tipos de características) que se cruzan, produciendo una descendencia, en la que los distintos rasgos de las distintos tipos de características ocurren en ciertas frecuencias relativas. Aquel pequeño subconjunto inicial de aplicaciones intencionales I(MEH) al cual se habría aplicado exitosamente la ley fundamental de MEH, así como también alguna de sus leyes especiales, constituiría el mencionado conjunto $\mathbf{I}(\mathbf{M E H})$ de aplicaciones (exitosas) paradigmáticas iniciales de tal teoría. Podría discutirse si dicha teoría ha sido aplicada exitosamente a los híbridos constantes del tipo de Hieracium, ya que la ley que rige el desarrollo/ evolución de tales híbridos fue sólo insinuada por Mendel. Lo que sí está fuera de toda duda es que las arvejas, género Pisum, investigadas por Mendel debieran ser consideradas como aplicaciones firmes o exitosas de $\mathbf{M E H}$, fungiendo desde un inicio como paradigmáticas.

El paradigma de MEH (T(MEH)) podría ahora ser caracterizado como sigue:

$\mathbf{T}(\mathbf{M E H}):=\left\langle\mathbf{K}_{0}(\mathbf{M E H}), \mathbf{I}_{e}(\mathbf{M E H})\right\rangle$ 


\section{III.2. LA TEORÍA DE MENDEL SOBRE LA FUNDAMENTACIÓN CELULAR DEL DESARROLLO/ EVOLUCIÓN DE LOS HÍBRIDOS (MFC)}

El marco conceptual de la teoría de Mendel sobre la fundamentación celular del desarrollo/evolución de los híbridos (la clase de sus modelos potenciales $\left.\mathbf{M}_{\mathbf{p}}(\mathbf{M F C})\right)$ se podría representar mediante estructuras como la siguiente: $\left\langle I,\left(C_{i}\right)\right.$ $\left.{ }_{i \leq k},\left(E_{i}\right)_{i \leq s}, A P P, M A T, D I S T,\left(D E T_{i}\right)_{i \leq k}, C O M B\right\rangle$, en donde I, APP, MAT y DIST representan lo mismo que en el caso de la teoría de Mendel sobre el desarrollo/ evolución de los híbridos $\mathbf{M E H}$, en tanto que $\left(E_{i}\right)_{i \leq s}$ representa el conjunto de tipos de elementos, $\left(D E T_{i}\right)_{i \leq k}$ una función que asigna características de cierto tipo a elementos de cierto tipo y COMB una función que representa la transición de elementos paternos a elementos en la descendencia.

Estructuras de este tipo constituyen extensiones teóricas de aquellas que permiten la representación de los sistemas a los cuales la teoría pretenden aplicarse (las aplicaciones intencionales I) y a los que se supone, hipotéticamente, que se han aplicado (las aplicaciones que, si fueran consideradas exitosas, incluirían a las paradigmáticas $\mathbf{I}_{e}$, tales como el comportamiento de los híbridos variables de las arvejas, género Pisum, y el comportamiento de los híbridos constantes de las hieracias, género Hieracium) caracterizable mediante las estructuras del tipo $y=\left\langle I,\left(C_{i}\right)_{i \leq k}, A P P, M A T, D I S T\right\rangle$, o, lo que es lo mismo, estas estructuras se obtienen a partir de aquéllas si les «recortamos» los componentes teóricos, es decir, son subestructuras parciales de ellas (y pertenecen a la clase de modelos parciales $\left.\mathbf{M}_{\mathrm{pp}}(\mathbf{M F C}):=\mathbf{r}\left(\mathbf{M}_{\mathrm{p}}\right)(\mathbf{M F C})\right)$.

Los distintos conceptos MFC-teóricos $\left(\left(E_{i}\right)_{i \leq s},\left(D E T_{i}\right)_{i \leq k}\right.$ y $\left.C O M B\right)$ contribuyen a explicar los sistemas empíricos conceptualizados mediante los conceptos MFC-no-teóricos $\left(I,\left(C_{i}\right)_{i \leq k},, A P P, M A T, D I S T\right)$ : la ampliación de los sistemas empíricos (de tipo $y=\left\langle I,\left(C_{i}\right)_{i \leq k}, A P P, M A T, D I S T\right\rangle$ ) mediante tales conceptos (tipos apropiados de elementos $\left(E_{i}\right)_{i \leq s}$, las relaciones $\left(D E T_{i}\right)_{i \leq k}$ en que éstos se encuentran con las características de distinto tipo de los individuos $I$ y su distribución en la descendencia (probabilidades esperadas o teóricas) $C O M B$ ) es tal que devienen modelos de MFC (M(MFC)), e.e. satisfacen la ley fundamental de MFC, la cual afirma que: existen elementos que difieren en su naturaleza y comportamiento («constitución y agrupamiento»), tales que las distribuciones (frecuencias relativas, proporciones) de características en la descendencia (DIST) concuerdan, exacta o aproximadamente, con las probabilidades postuladas de distribución de los elementos $(C O M B)$, dadas ciertas relaciones, igualmente postuladas, entre las características y dichos elementos $\left(D E T_{i}\right)_{i \leq k}$.

Las condiciones de ligadura de MFC (C(MFC)) establecen relaciones del tipo de las denominadas de igualdad. Una de ellas establece la exigencia de que a los mismos elementos les sean asignados las mismas características en todas las aplicaciones de la teoría en que ellos ocurran, o sea, es una condición de 
ligadura sobre la función $\left(D E T_{i}\right)_{i \leq k}$ (en símbolos: $\mathbf{C}_{D E T}^{\square,=\square}$. La otra establece que los mismos elementos parentales se distribuyen en la descendencia siempre de la misma manera en todas las aplicaciones de la teoría en que ellos ocurran, o sea, es una condición de ligadura sobre la función $C O M B$ (en símbolos: $\mathbf{C}_{C O M B}^{\square}$ ). La condición de ligadura global de $\mathbf{M F C}(\mathbf{C}(\mathbf{M F C}))$ es la intersección de todas las condiciones de ligadura de $\mathbf{M}_{\mathbf{p}}(\mathbf{M F C}): \mathbf{C}(\mathbf{M F C}):=\mathbf{C}_{D E T}^{\square,=\square} \cap \mathbf{C}_{C O M B}^{\square,=\square}$.

Los vínculos que MFC tiene con otras teorías (pre-teorías, proto-teorías, «folk-theories») subyacentes, presupuestas, por medio de las cuales se determina la extensión de aquellos conceptos que son MFC-no-teóricos, o sea, de los conceptos simbolizados como $I,\left(C_{i}\right)_{i \leq k}, A P P, M A T$ y DIST, son los mismos que los señalados para el caso de MEH. El vínculo interteórico global de MFC (L(MFC)), formado por la intersección de todos vínculos interteóricos que tiene esta teoría con otras teorías subyacentes, presupuestas, se define de la misma manera que para el caso de MEH: $\mathbf{L}(\mathbf{M F C}):=\cap\left\{\lambda_{1}, \lambda_{2}, \lambda_{3}, \lambda_{4}\right\}$.

Ahora estamos en condiciones de caracterizar el núcleo teórico de MFC $\left(\mathbf{K}_{0}(\mathbf{M F C})\right)$ como sigue:

$$
\mathbf{K}_{0}(\mathrm{MFC}):=\left\langle\mathbf{M}_{\mathrm{p}}(\mathrm{MFC}), \mathbf{M}(\mathrm{MFC}), \mathbf{M}_{\mathrm{pp}}(\mathrm{MFC}), \mathbf{C}(\mathrm{MFC}), \mathbf{L}(\mathrm{MFC})\right\rangle
$$

El dominio de aplicaciones intencionales de MFC está constituido por la clase de aquellos sistemas empíricos a los que uno desea aplicar su ley fundamental. Desde un punto de vista formal podemos decir que $\mathbf{I}(\mathbf{M F C}) \subseteq \mathbf{M}_{\mathrm{pp}}(\mathbf{M F C})$ y que los miembros de I(MFC) son sistemas empíricos que contienen individuos (individuos propiamente dichos o poblaciones) con una cierta apariencia (es decir, con ciertos rasgos o tipos de características) que se cruzan, produciendo una descendencia, en la que los distintos rasgos de las distintos tipos de características ocurren en ciertas frecuencias relativas. Aquel pequeño subconjunto inicial de aplicaciones intencionales I(MEH) al cual se habría aplicado exitosamente la ley fundamental de MEH constituiría el mencionado conjunto $\mathbf{I}_{e}(\mathbf{M E H})$ de aplicaciones (exitosas) paradigmáticas iniciales de tal teoría. Aunque a la explicación del comportamiento de los híbridos variables tales como Pisum, basada en una unión pasajera de los elementos celulares diferentes, y del comportamiento de los híbridos constantes tales como Hieracium, basada en una unión duradera de los elementos celulares diferentes, «puede naturalmente atribuírsele sólo el valor de una hipótesis», si fueran aplicaciones firmes o exitosas de MFC, serían sin duda sus aplicaciones paradigmáticas.

El paradigma de MFC (T(MFC)) podría ahora ser caracterizado como sigue:

$$
\mathbf{T}(\mathbf{M F C}):=\left\langle\mathbf{K}_{0}(\mathbf{M F C}), \mathbf{I}_{e}(\mathbf{M F C})\right\rangle
$$




\section{Preguntas en el hibridismo de Mendel}

Ahora aplicaremos la propuesta erotético-teórica al caso del hibridismo de Mendel.

Para dar respuesta a la pregunta «icómo se desarrollan/evolucionan los híbridos?», que llevaría a decidir acerca de la cuestión de si pueden originarse nuevas especies a partir del cruzamiento de especies preexistentes, Mendel propone MEH.

Si tomamos en cuenta lo dicho en la sección II acerca del tratamiento estructuralista de las restricciones de los problemas, podemos decir que la pregunta completa para la generación (conformada por el propio Mendel) de científicos que aplica $\mathbf{T}(\mathbf{M E H}):=\left\langle\mathbf{K}_{0}(\mathbf{M E H}), \mathbf{I}_{e}(\mathbf{M E H})\right\rangle$ en el tiempo $t$ tiene la forma: $Q=\left\langle(? p) q(p),\left\langle\mathbf{K}_{0}(\mathbf{M E H}), \mathbf{I}_{e}(\mathbf{M E H})\right\rangle\right\rangle$, en donde $(? p) q(p)$ es «¿cómo se desarrollan/evolucionan los híbridos?» (en donde dicho desarrollo/evolución puede ser concebido mediante la distribución de las características en la descendencia de los híbridos) y $\left\langle\mathbf{K}_{0}(\mathbf{M E H}), \mathbf{I}_{e}(\mathbf{M E H})\right\rangle$ el paradigma de $\mathbf{M E H}$ que utiliza la generación de hibridistas (Mendel) en el tiempo $t$ y que restringe el conjunto de respuestas admisibles.

Recordemos, además, que la noción de teoría convierte a las preguntas «cómo» iniciales de las teorías fenomenológicas como la teoría en cuestión en preguntas «wh-» más manejables y en «pequeñas» preguntas «sí-no». Una interrogación que comienza como una pregunta «cómo», formulada en el lenguaje-en-uso de la comunidad de hibridistas, y concerniente a un área de experiencia («¿cómo se desarrollan/evolucionan los híbridos?», e. e. «¿cómo se distribuyen las características en la descendencia de los híbridos?»), se torna (en el estado «paradigmático» en el que hay un núcleo conceptual $\mathbf{K}_{0}(\mathbf{M E H})$ con su vocabulario) en una pregunta «qué» («iqué leyes especiales (condiciones de ligadura, vínculos interteóricos) se necesitan, dentro de los estándares impuestos por $\mathbf{T}(\mathbf{M E H})$, para transformar en hechos conocidos una aplicación propuesta no examinada hasta ahora o recalcitrante?») o «cuál» («¿cuál es la ley especial que rige este particular conjunto de aplicaciones $\mathbf{I}_{i}(\mathbf{M E H})$ ?»). Posteriormente, se encuentran preguntas «sí-no» («ies ésta la ley especial que rige este particular conjunto de aplicaciones $\mathbf{I}_{i}(\mathbf{M E H})$ ?»).

$\mathrm{Y}$ es en este punto en que Mendel formula, en primera instancia, «la ley de desarrollo/evolución encontrada en Pisum», es decir, «la ley de la combinación simple de las características» y su generalización, «la ley de la combinación de las características diferenciales», que valdría para los híbridos variables, para luego intentar formular una ley sobre el desarrollo/evolución que valiera para los híbridos constantes, considerando ambas leyes como leyes especiales de una ley más general, la ley fundamental de la teoría, que Mendel nunca llegó a enunciar. 
Por otro lado, intenta fundamentar y explicar «la ley de desarrollo/evolución encontrada en Pisum», que rige el comportamiento de los híbridos variables, así como también el comportamiento de los híbridos constantes, mediante la relación entre la producción y comportamiento de las células germinales y polínicas y el de las formas (o características) constantes y, en última instancia, en la naturaleza y comportamiento («constitución y agrupamiento») de lo que denomina «elementos» $\mathrm{o}$ «elementos celulares». Para ello, propone, aun cuando sólo a título hipotético, MFC.

La pregunta completa para la generación de científicos (conformada por el propio Mendel) que aplica $\mathbf{T}(\mathbf{M F C}):=\left\langle\mathbf{K}_{0}(\mathbf{M F C}), \mathbf{I}_{e}(\mathbf{M F C})\right\rangle$ en el tiempo $t$ tiene la forma: $Q=\left\langle(? p) q(p),\left\langle\mathbf{K}_{0}(\mathbf{M F C}), \mathbf{I}_{e}(\mathbf{M F C})\right\rangle\right\rangle$, en donde $(? p) q(p)$ es «ipor qué los híbridos se desarrollan/evolucionan del modo en que lo hacen?» (en donde dicho desarrollo/evolución puede ser concebido mediante la distribución de las características en la descendencia de los híbridos) y $\left\langle\mathbf{K}_{0}(\mathbf{M F C}), \mathbf{I}_{e}(\mathbf{M F C})\right\rangle$ el paradigma de MFC que utiliza la generación de hibridistas (Mendel) en el tiempo $t$ y que restringe el conjunto de respuestas admisibles.

Nuevamente, recordemos, además, que la noción de teoría convierte a las preguntas «porqué» iniciales en preguntas «wh-» más manejables y en «pequeñas» preguntas «sí-no». Una interrogación que comienza como una pregunta «porqué», formulada en el lenguaje-en-uso de la comunidad de hibridistas, y concerniente a un área de experiencia («ipor qué los híbridos se desarrollan/ evolucionan del modo en que lo hacen?», e. e. «ipor qué se distribuyen las características en la descendencia de los híbridos del modo en que lo hacen?»), se torna (en el estado «paradigmático» en el que hay un núcleo conceptual $\mathbf{K}_{0}(\mathbf{M F C})$ con su vocabulario) en una pregunta «qué» («iqué leyes especiales (condiciones de ligadura, vínculos interteóricos) se necesitan, dentro de los estándares impuestos por T(MFC), para transformar en hechos conocidos una aplicación propuesta no examinada hasta ahora o recalcitrante?») o «cuál» («icuál es la ley especial que rige este particular conjunto de aplicaciones $\mathbf{I}_{i}(\mathbf{M F C})$ ?»). Posteriormente, se encuentran preguntas «sí-no» («¿es ésta la ley especial que rige este particular conjunto de aplicaciones $\mathbf{I}_{i}(\mathbf{M F C})$ ?»).

Mendel responde la cuestión «¿cuál es la ley especial que rige este particular conjunto de aplicaciones $\mathbf{I}_{i}(\mathbf{M F C})$ ?» haciendo hipótesis acerca de: 1) los tipos de elementos, 2) su relación con las características (la forma matemática concreta asumida por $D E T$ ), y 3) su distribución entre los individuos en la descendencia (la forma matemática concreta asumida por $C O M B$ ).

Una vez realizadas las hipótesis particulares acerca de cada uno de tales aspectos, p.e., para el caso de híbridos variables en donde se consideren dos características diferenciales con distribución 3:1, si se postula que 1) bastan dos tipos de elementos, 2) un tipo de elementos es dominante y el otro recesivo, y 3) se distribuyen con igual probabilidad, la pregunta anterior (pregunta «wh-» 
-«cuál»-más manejable) se convierte en una pregunta más pequeña («¿es ésta la ley especial que rige este particular conjunto de aplicaciones $\mathbf{I}_{i}(\mathbf{M F C})$ ?») que podemos responder por sí o por no.

Hipótesis similares se propondrían para tratar el caso de híbridos variables en donde se consideren cuatro características diferenciales con distribución 9:3:3:1 y, ligereamente diferentes, para el caso de los híbridos constantes. Tanto en un caso como en el otro la pregunta inicial se convierte en una pregunta más pequeña («ies ésta la ley especial que rige este particular conjunto de aplicaciones $\mathbf{I}_{i}(\mathbf{M F C})$ ?») que podemos responder por sí o por no.

$\mathrm{Si}$ ahora comparamos las preguntas que se formulan e intentan responder y/o los problemas que se plantean e intentan resolver en el hibridismo de Mendel, podemos decir lo siguiente.

Las distintas preguntas que se formulan y los problemas que se plantean dentro del marco de cada uno de los respectivos paradigmas -el de MEH y el de $\mathbf{M F C}$ - constituyen, en todo caso, reformulaciones más precisas de uno y el mismo problema, perteneciendo así al primer tipo de cambio problemático identificado en la sección II o «cambio en un problema».

Sin embargo, las preguntas que se formulan y los problemas que se plantean en MEH son claramente distintos de aquellos que son formulados y planteados en MFC: tanto los enunciados interrogativos como las preguntas completas, dadas no sólo por dichos enunciados, sino también por los respectivos paradigmas, son diferentes.

Es así que, en el paso de MEH a MFC, nos encontramos frente al segundo tipo de cambio problemático identificado en la sección II o «cambio de problemas».

\section{CONCLUSIONES}

En este trabajo se intentó mostrar que la oposición entre el enfoque de resolución de problemas y el enfoque de teorías es sólo aparente, ya que, según se vio, los problemas solamente pueden ser identificados sobre la base de teorías y, más aún, los problemas heredan su estructura de las teorías, que los refinan y dan una guía heurística en la búsqueda de respuestas.

Más aún, vimos que el estructuralismo metateórico puede precisar el enfoque de resolución de problemas, ayudando a proporcionar un criterio de identidad para preguntas, pues la estructura de los problemas refleja la estructura del elemento teórico básico y de la red teórica de la que ellos descienden, y, así, contribuyendo también al análisis de la historia de los problemas (y sus respuestas), permitiendo distinguir entre dos tipos de cambios problemáticos: «cambios en un problema» $\mathrm{y}$ «cambios de problema».

Esto se hizo a partir de una propuesta erotético-teórica, basada en la que efectúa Sintonen $(1985,1996)$-quien sigue análisis y sugerencias de Tuomela 
(1980)- y en ciertas ideas de Hintikka (1981), y de su aplicación al caso de la historia de la genética, pudiendo identificar, relacionar y distinguir los problemas a los que se han enfrentado el «hibridismo» de Johann Gregor Mendel, con sus dos partes: la «teoría de Mendel sobre el desarrollo/evolución de los híbridos» y la «teoría de Mendel sobre la fundamentación celular del desarrollo/evolución de los híbridos».

\section{REFERENCIAS BIBLIOGRÁFICAS}

BALZER, W., MOULINES, C. U. y J. D. SNEED (1987): An Architectonic for Science. The Structuralist Program. Dordrecht: Reidel. Tr. Pablo Lorenzano, Una arquitectónica para la ciencia. El programa estructuralista. Bernal: Universidad Nacional de Quilmes, 2012.

BELNAP, N. D. y T. B. STEEL (1976): The Logic of Questions and Answers. New Haven and London: Yale University Press.

CASANUEVA, M. (2002): «La red teórica de la hibridación mendeliana», en J. A. Díez y P. Lorenzano (eds.), Desarrollos actuales de la metateoría estructuralista: problemas y discusiones. Quilmes: Universidad Nacional de Quilmes/Universidad Autónoma de Zacatecas /Universidad Rovira i Virgili, pp. 231-261.

HINTIKKA, J. (1981): «On the Logic of an Interrogative Model of Scientific Inquiry». Synthese, 47, 1, pp. 69-83.

LORENZANO, P. (1995): Geschichte und Struktur der klassischen Genetik. Frankfurt am Main: Peter Lang.

, (1997): «Hacia una nueva interpretación de la obra de Mendel», en J. Ahumada y P. Morey (eds.), Selección de trabajos de las VII Jornadas de Epistemología e Historia de la Ciencia. Córdoba: Facultad de Filosofía y Humanidades, Universidad Nacional de Córdoba, pp. 220-231.

LORENZANO, P. (2011): «What Would Have Happened if Darwin Had Known Mendel (or Mendel's Work)?», History and Philosophy of the Life Sciences, 33, pp. 3-48.

MENDEL, G. (1865): «Versuche über Pflanzen-Hybriden», Verhandlungen des Naturforschenden Vereins zu Brünn 4, pp. 3-57, reimpreso en Ostwalds Klassikern der exakten Wissenschaften, Nr. 6, Braunschweig: Friedr. Vieweg \& Sohn, 1970.

, (1866): «Brief an Carl Nägeli vom 31.12.1866», en C. Correns (ed.), «Gregor Mendels Briefe an Carl Nägeli, 1866-1873. Ein Nachtrag zu den veröffentlichten Bastardierungsversuchen Mendels», Abhandlungen der mathematisch-physischen Klasse der Königlich Sächsischen Gesellschaft der Wissenschaften, 29 (1905), pp. 1237-1240. 
, (1869): «Über einige aus künstlicher Befruchtung gewonnenen HieraciumBastarde», Verhandlungen des Naturforschenden Vereins zu Brünn 8, pp. 2631.

, (1870): «Brief an Carl Nägeli vom 03.07.1870», en C. Correns (ed.), «Gregor Mendels Briefe an Carl Nägeli, 1866-1873. Ein Nachtrag zu den veröffentlichten Bastardierungsversuchen Mendels», Abhandlungen der mathematisch-physischen Klasse der Königlich Sächsischen Gesellschaft der Wissenschaften, 29 (1905), pp. 1266-1273.

MOULINES, C. U. (1991): Pluralidad y recursión. Madrid: Alianza, 1991.

SINTONEN, M. (1985): «Separating Problems from their Backgrounds: a Questiontheoretic Proposal», en J. Hintikka y F. Vandamme (eds.), Logic of Discovery and Logic of Discourse. New York: Plenum Press, pp. 25-49.

SINTONEN, M. (1996): «Structuralism and the Interrogative Model of Inquiry», en Balzer, W. y C. U. Moulines (eds.), Structuralist Theory of Science: Focal Issues, New Results. Berlin: de Gruyter, pp. 45-74.

TUOMELA, R. (1980): «Explaining Explaining», Erkenntnis, 15, 2, pp. 211-243.

Pablo Lorenzano es Profesor Titular Ordinario, Categoría «A» en la Universidad Nacional de Quilmes (UNQ)/ Consejo Nacional de Investigaciones Científicas y Técnicas (CONICET) (Argentina).

Líneas de investigación:

Filosofía General de la Ciencia, filosofía de la biología, historia de la biología, historia de la filosofía de la ciencia

Publicaciones recientes:

(2012) «Estructuras y aplicaciones intencionales: inconmensurabilidad teórica y comparabilidad empírica en la historia de la genética clásica», en Lorenzano, P. y O. Nudler (eds.), El camino desde Kuhn. La inconmensurabilidad hoy, Madrid: Editorial Biblioteca Nueva, pp. 289-350.

(2012) «Who Got What Wrong? Sober and F\&PP on Darwin: Guiding Principles and Explanatory Models in Natural Selection» (escrito con José A. Díez), Erkenntnis, DOI: 10.1007/ s10670-012-9414-3, Online First Articles.

Direcciónelectrónica: pablol@unq.edu.ar 